\title{
Contributions of different feedback assistance to text memorial representation
}

\author{
PHILIP LANGER, VERNE KEENAN, and JASON BERGMAN \\ University of Colorado, Boulder, Colorado
}

\begin{abstract}
Subjects were assigned to read one of two descriptions of a town. The route version was based on the town as a driver would encounter it, while the survey version provided a spatial description. Three feedback conditions were provided: (1) limited access to a map, (2) limited review of previously read sentences, and (3) an opportunity to read the entire text after processing. There were no differences in amount of material recalled or discrimination between old text and paraphrases, but feedback differentially assisted inferential reasoning. Feedback that supplemented the version provided proved more efficacious.
\end{abstract}

One of the more ubiquitous beliefs among both researchers and practitioners in teaching concerns the supposed efficacy of instructional assistance, particularly feedback (Berliner \& Rosenshine, 1977). For example, even while Kulhavy (1977) was demolishing a number of sacred and simplistic assumptions regarding feedback, he still concluded that it should be given as often as possible. Our own research, however (Langer, Keenan, \& Cumbo, 1992), suggests that insofar as text comprehension is concerned, feedback is not always that effective.

Originally, we axiomatically accepted the premise that feedback was an instructional necessity. Therefore our efforts were initially directed toward estimating the contributions of feedback to the comprehension of reconstructed text. Subsequently, we expanded our concept of assistance to include such devices as signaling (Langer, Keenan, \& Culler, 1989). However, the inconsistency of our findings led us to conclude that the belief that "any help is better than no help" is not true. College students seemed to do rather well in reconstructing text of varying degrees of familiarity with or without external assistance. Indeed our initial interest in feedback subsequently resolved itself into two related issues: feedback and text comprehension (Langer, Keenan, \& Nelson, 1991). This expansion of the problem is not very common, however. Reviews of the literature in educational research indicate that insofar as teaching is concerned, studies have generally been focused on the contributions of feedback to reading skills and outcomes (Moore, 1988). Absent has been any idea of a particular model of text comprehension. As we shall point out shortly, this is a serious deficit.

Feedback is usually defined as any form of assistance subsequent to a response, designed to inform or correct student performance (Langer \& Keenan, 1984). The feedback may range from a rather simple yes/no, to assistance

The present report is based in part on a paper presented at the meeting of the Psychonomic Society, St. Louis, in November 1992. Requests for reprints should be sent to P. Langer, Psychology Department, CB 345, University of Colorado, Boulder, CO 80309. that not only provides the correct answer, but also directs the student to engage in self-corrective behavior (Andre $\&$ Theiman, 1988). However, the more complex feedback procedures may become instructional tasks in themselves (Kulhavy, 1977). The form of feedback itself is another issue. Holding (1965) pointed out that the feedback provided may vary along any number of dimensions such as type, timing, density (i.e., constant or intermittent), and so forth. The number of permutations and combinations is awesome. To make matters even more interesting, in an analysis of the contributions of feedback to concept discrimination, estimates of effect sizes for interactions were greater than those for principal effects (Getsie, Langer, \& Glass, 1985). Indeed, our own research efforts in recent years have been focused on interaction effects (Langer et al., 1992).

The question of whether feedback is motivational or an information provider seems to have died down. The original assumptions that categorized feedback as reinforcement were a product of the development of programmed instruction within a behavioristic framework (Kulhavy, 1977; Langer et al., 1992). However, an accumulation of research findings suggesting that praise for a correct response (i.e., reward) was not as effective a contributor to learning as was being informed of an incorrect response (i.e., punishment) seems to have drawn the issue to a close (Barringer \& Gholson, 1979; Getsie et al., 1985). Of course, it also seems evident to us that if feedback primarily provides information, the information must bear some relation to not only what is to be learned but how the material is being processed. Unfortunately, the latter issue has not always been considered. That is, arguing for the success of feedback based solely on some set of outcomes can be misleading.

Andre and Theiman (1988), for example, found differences in the relative contributions of feedback of adjunct questions in terms of concept acquisition as opposed to learning facts. Indeed all too often experiments designed to estimate the effects of feedback have required a factual level response. The early and persistent influence of 
behaviorism contributed to the problem, insofar as an associationist model was accepted as a given. This model led researchers to decompose more complex learning into small, verifiable units, generally at a level related to facts (Kulhavy \& Anderson, 1972).

On the other hand, the absence of any systematic employment of text comprehension models is also a serious concern. If anything, typical educational designs involving such activities as the study of reciprocal teaching on text comprehension (Moore, 1988) ignore how the information is being processed and stored. As Kulhavy (1977) pointed out, students do not necessarily come to every instructional event completely naive regarding content. Thus, assistance in the form of generic instructional processes can only lead to eventual incorporation of superstitious procedural behaviors, especially if there is some positive effect.

In our work, we have incorporated Kintsch's (1988) construction-integration paradigm and categories of universal representation (Kintsch, Welch, Schmalhofer, \& Zimny, 1990). Our data led us to acknowledge that unless feedback is tied to some model of how the assistance fits into ongoing text manipulation, there is little hope of deriving any meaningful relation between help and comprehension (Langer et al., 1992). Kintsch (1988) argued that the initial text propositions are formed directly from the text itself but are then elaborated and integrated into a coherent text-based representation, guided by the prior knowledge available. Clearly this is an iterative process in which new text to be incorporated is checked against the current text base. The model suggests that several layers of knowledge interact simultaneously in the task of understanding discourse. Evidence for the model may show a surface representation of verbatim text, a propositional representation of semantic meanings, and a situation representation of pragmatic interpretations. Linguistic elements and syntactic chunks (surface representation features) interact with propositions (semantic representations) and situation elements (Kintsch et al., 1990).

The situation representation was tested in a series of experiments by Perrig and Kintsch (1985). In that investigation, there were two descriptions of a mythical town called Baldwin. One version was composed in terms of a sequential set of instructions for driving through the town, and the other in the context of a spatial or geographic layout. Surface representation was detected by recognition of verbatim sentences, text-based representation was detected by propositional recall, and situation representation was assessed by inferences.

This study is the fourth in a series based on Perrig and Kintsch's (1985) work. The current research differs from our earlier work in several important ways: (1) The text is presented in appropriate canonical order rather than scrambled, (2) several different feedback types are used, and (3) feedback in these studies is defined in terms of assistance given in response to an overt request for aid, with the assumption that it is influencing current processing in a manner similar to hypothesis testing. The origi- nal description of the town has been slightly modified, so we chose to call our town Mapleton. As in Perrig and Kintsch's research, there are two descriptive versions of the town, each 25 sentences long. One is a spatial or survey description, while the other is a route version that guides the driver through the town. The stories were typed one sentence to a card and read aloud one at a time. This was designed to control the reading process. The feedback provided was, as in previous studies, a map of Mapleton, a chance to review previously read text during the processing, or an opportunity to read the complete text at the end of the processing. We uncovered in our third study a statistically significant version $\times$ feedback interaction, which we decided was worth pursuing. Such findings of interactions have been limited in previous work. There are, however, two differences in the present study: (1) In order to enhance recall scores, which have been quite low in the past, all three feedback groups read the complete text after the feedback conditions, and (2) in order to provide a common direction orientation for both versions, for the route version one sentence provided a specific reference to direction, and the map indicated north.

\section{METHOD}

Our subjects were $\mathbf{4 2}$ general psychology students. As in the past, the sentences were printed one to a card, and the subjects read each card aloud. Three feedback conditions were provided, as follows: (1) In the map condition, the subjects, after reading a sentence, were allowed to see a schematic map of Mapleton for up to $10 \mathrm{sec}$. They could make five such requests, totaling $50 \mathrm{sec}$. (2) In the sentence condition, the subjects, after reading a sentence, could study a sentence they had just read for an additional $10 \mathrm{sec}$. They could make five such requests, totaling $50 \mathrm{sec}$. (3) In the text condition, after reading the 25 cards, the subjects had the complete text made available, which they could study for $50 \mathrm{sec}$. Then all groups read the complete text. This gave us a six-group comparison, based on a two versions (route/survey) $\times$ three feedbacks (map/sentence/text) design.

So that we could assess semantic representation, after reading the cards the subjects were first asked to write down what they remembered. This recall protocol was scored for both number of propositions and order of report. Upon completion of the protocol, the subjects were presented with a 32-card deck, consisting of old sentences from both versions as well as new sentences. The latter were paraphrases written in either the route or the survey style, and they could be either true or false inferences.

Situation memory was determined as follows: The subjects read each sentence aloud and determined whether the sentence was "true" or "false." True and false cards were placed in separate piles. It follows that an original sentence from either version is a true inference, by definition, whereas paraphrases, as previously noted, could be either true or false.

To assess surface representation, the subjects then went through the "true" pile and indicated which were "old" sentences. This recognition measure constituted our index of surface memory-that is, verbatim sentence recognition. Obviously, an original sentence from the other version as well as a paraphrase written in either version style could be seen as true, but could not appropriately be classified as old, given the version the subjects had previously read.

\section{RESULTS}

Again, following Perrig and Kintsch's (1985) previous work, we measured three dimensions of memory. Seman- 
tic memory was assessed by total number of propositions recalled, as well as the agreement of recall order with original order (tau). Discrimination between old and new sentences measured surface memory, and situation memory was assessed by inferential reasoning. The $d^{\prime}$ statistic was used for our analyses of surface and situation memory.

Neither the main effects nor the interactions for recall and recognition were statistically significant, nor were they even very interesting. Yet there were trends consistent with previous studies. For example, propositional recall (i.e., semantic memory) was marginally superior for the route version (44.20) in comparison with survey (39.29). The sentence (44.83) and text (43.14) feedback conditions were marginally superior to map feedback (36.57). For recognition, the differences were all statistically nonsignificant. The route and survey mean differences were hardly distinguishable, whereas among the feedback conditions, text was marginally superior.

Our assessment of inferential reasoning created some problems. Initially, we decided to separately analyze inferential reasoning on the basis of the version read as distinct from the version not read. The reader will recall that subjects reading either version used a common set of mixed statements. For inferential reasoning, again, there were old and new sentences from both versions. The first results yielded negative $d^{\prime}$ 's, which are rather difficult to explain. However, an item analysis revealed that a number of the paraphrases were more in the nature of trick- item test questions, so we decided to eliminate the four worst offenders, two from each version. We then combined all inferences, and the following discussion is based on those modifications.

Although the findings for inferential reasoning were also not statistically significant, the interactions were reliable and in a direction we have seen earlier (Langer et al., 1992). Map feedback (.48) was superior to text (.04) for the route version and only marginally lower than sentence (.56) feedback. In contrast, contributions in the sentence (.67) and text (.43) feedback conditions increased for the survey version, whereas the map (.07) contributed very little. It seemed to us that the spatial information provided by the map added to an understanding of the route version, which is basically a verbally guided tour of the town, whereas the verbal feedback provided by the sentence and text feedback conditions supplemented the spatial or geographic description of Mapleton. Figure 1 shows the essentially parallel gains for both the text and the sentence feedback conditions, while map assistance decreases dramatically.

\section{DISCUSSION}

Aside from the experimental findings that we will discuss in a moment, and unlike many of our colleagues in educational research, we believe that valuable information regarding text processing can be obtained within a controlled laboratory setting. From an instructional point of view, it would appear that assistance in this study was effective in

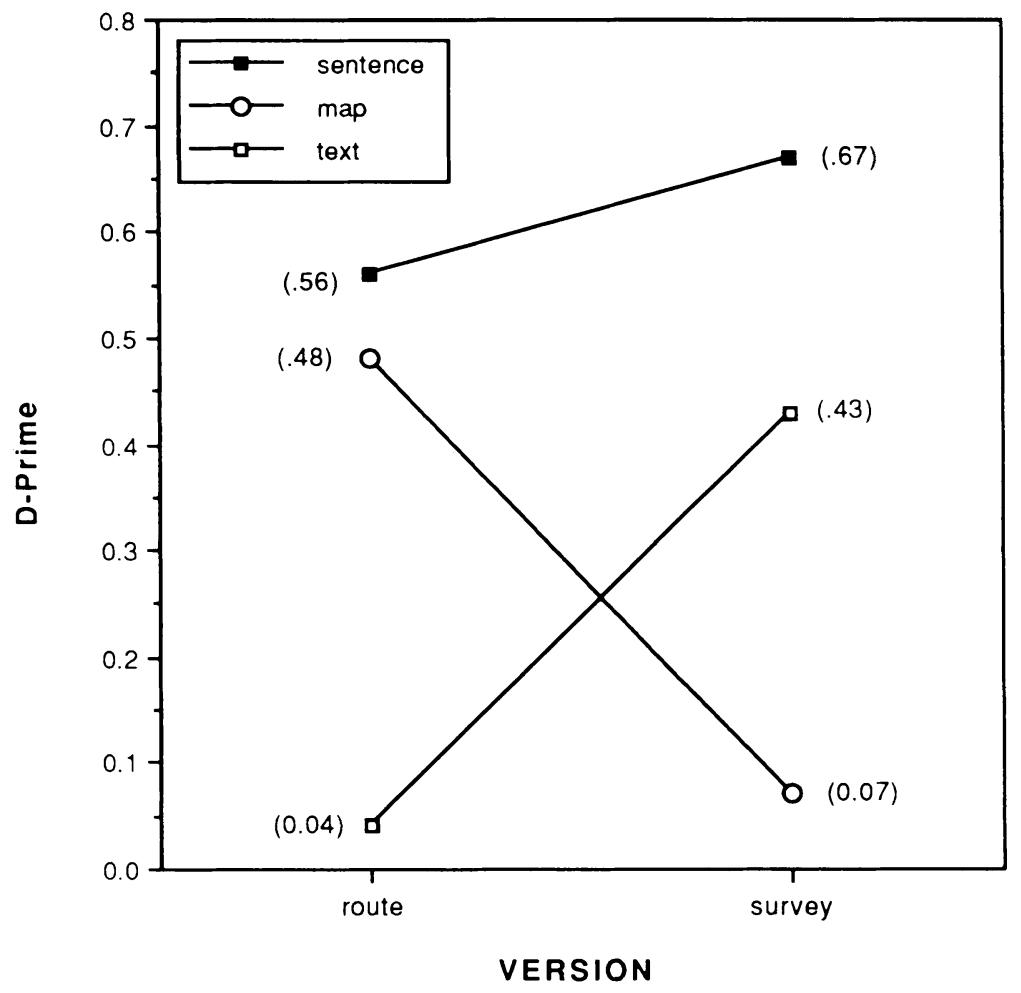

Figure 1. Route and survey versions $\times$ map, sentence, and text feedback interactions for selected data. 
providing supplemental information to assist processing for the text provided. There is little reason to suspect that feedback served basically a corrective function. Again, when subjects request assistance, we assume that it is the outcome of a direct decision to test certain assumptions about understanding. Indeed we have argued in the past that text processing is not too dissimilar from problem solving, insofar as it involves testing hypotheses. Currently we treat such requests as actual decision points in the processing, with feedback providing the needed supplemental information.

Again, in Kintsch's (1988) model and in Kulhavy's (1977) work, there is the clear recognition that information possessed or obtained by the reader modifies subsequent processing. For example, early in our own research (Langer, Keenan, \& Medosch-Schonbeck, 1985), we found that subjects were able to get the gist of scrambled discourse even though comparatively few actually knew much about the historical referent, which in this case was the development of the atom bomb at Chicago by Enrico Fermi. That is, they could tell us it was about the atom bomb or World War II, but not much more. It could be argued, of course, that college students may have mastered the recall of little-understood materials to an art form.

Nevertheless, we believe that what students have learned, or are currently acquiring, must be reviewed in the context of some model of processing. While there are any number of models of reading comprehension available (e.g. Britton \& Black, 1985), failure to incorporate any systematic analysis of how text is processed can lead to the indiscriminate use of adjunct aids, feedback being the first choice. We continue to believe that assistance must be based on assumptions about the learning processes involved rather than a set of a priori principles of management based on too-often low-level outcomes.

\section{REFERENCES}

ANDre, T., \& Theiman, A. (1988). Level of adjunct question, type of feedback, and learning concepts by reading. Contemporary Educational Psychology, 13, 296-307.

BARRINGer, C., \& GHOLSON, B. (1979). Effects of type and combination of feedback upon conceptual learning of children: Implications for research in academic learning. Review of Educational Research, 49, 459-478.
BERLINER, D. C., \& Rosenshine, B. (1977). The acquisition of knowledge in the classroom. In R. C. Anderson, R. J. Spiro, \& W. E. Montague (Eds.), Schooling and the acquisition of knowledge (pp. 375404). Hillsdale, NJ: Erlbaum.

Britton, B. K., \& BLACK, J. B. (1985). Understanding expository text. Hillsdale, NJ: Erlbaum.

Getsie, R., Langer, P., \& Glass, G. V. (1985). Meta-analysis of the effects of type and combinations of feedback on children's discrimination learning. Review of Educational Research, 55, 9-22.

Holding, D. H. (1965). Principles of learning. New York: Oxford University Press.

KINTSCH, W. (1988). The role of knowledge in discourse comprehension: A construction-integration model. Psychological Review, 95, 163-182.

Kintsch, W., Welch, D., Schmalhofer, F., \& Zimny, S. (1990). Sentence memory: A theoretical analysis. Journal of Memory \& Language, 29, 133-159.

Kulhavy, R. W. (1977). Feedback in written instruction. Review of Educational Research, 47, 211-232.

Kulhavy, R. W., \& ANDERSON, R. C. (1972). Delay-retention effects with multiple-choice tests. Journal of Educational Psychology, 65, 503-512.

Langer, P., \& Keenan, V. (1984). Modification of current feedback strategies: A text synthesis approach (Tech. Rep. No. 127). Boulder: University of Colorado, Institute of Cognitive Science.

Langer, P., Keenan, V., \& Culler, J. (1989). Text cueing and comprehension. Psychological Reports, 64, 1147-1158.

Langer, P., Keenan, V., \& Cumbo, K. (1992). Effects of text version and feedback type on memorial representations. Bulletin of the Psychonomic Society, 30, 373-376.

Langer, P., Keenan, V., \& Medosch-Schonbeck, C. (1985). A text synthesis approach to feedback. Psychological Reports, 57, 259-268.

Langer, P., Keenan, V., \& Nelson, S. (1991). Sentence-order feedback during processing of sequential or spatial texts. Bulletin of the Psychonomic Society, 29, 31-32.

Moore, P. (1988). Reciprocal teaching and reading comprehension: A review. Journal of Research in Reading, 11, 3-14.

Perrig, W., \& Kintsch, W. (1985). Propositional and situational representation of text. Journal of Memory \& Language, 24, 503-518.

(Manuscript received December 26, 1992.) 